\title{
The Effect of Disease and Harvest in the Eco-Epidemical Food Chain Model
}

\author{
Rasha Majeed Yaseen \\ Department of Mechatronics, Al-Khwarizmi College of Engineering, University of Baghdad-Iraq. \\ E-mail: rasha.majeed1@gmail.com.
}

\begin{abstract}
This paper describes a three species food web model with linear functional response and incidence disease. This model consisting of a prey, intermediate predator and top predator where harvesting of top predator species and SIS disease spread in prey are taken into consideration. The stability analysis of all possible equilibrium points are carried out. We discussed the effect both of harvest and disease on the stability of this model. Finally, we used the numerical simulations to verify the analytical results.
\end{abstract}

Keywords: SIS epidemics disease, prey-Predator model, Chain of food, Harvest management; stability.

\section{Introduction}

Mathematics is one way to explain many of the ideas and concepts in the sciences. In the field of food webs play a very important role in ecology, a lot of theoretical studies were carried out since the beginning of last century to explain the interaction between the ecological communities. One particular study describes the interaction between one population (prey) and the other (predator) living in a closed environment with the three populations striving for survival, for example see [1-6]. On the other hand densely populated areas are a good incubator for the spread of infectious diseases. Therefore, there is increasing opportunity for the spread of diseases among the communities interacting with each other [7-18]. There are numerous studies on the effects of harvesting on population growth. In the context of predatorprey interaction, some studies that treat the populations being harvested as a homogeneous resource include those [19-26]. In this paper, we proposed and analyzed a three species food web model in which the prey follows the susceptible-infectedsusceptible cycle and the top predator is harvested. In this model, we used linear form as a functional response and disease incidence for describing the transition of diseases. To be followed by a study on the stability of the equilibrium points. Next, we discuss the nature of the solutions and finally the numerical simulations to support the model.

\section{Mathematical Model}

We consider the following system as a model simulating a tritophic level food chain. The dynamics of three species food chain model with linear type of function response is governed by the following differential equation, where $N(T)$ is the population density of the lowest trophic level species (prey) at time $T$, there is SIS (SusceptibleInfected-Susceptible) epidemic disease spread among the prey population and it transmitted between the prey individuals by contact, according to linear incidence rate with infection rate constant $h_{3}>0$. The infected prey can be recovered and become susceptible again with recovery rate constant $h_{5}>0$. Therefore, the total prey population is divided into two classes: susceptible individuals that is denoted by $S(T)$, and infected individuals that is denoted by $I(T)$. Hence at any time $T$ the total prey population is $N(T)=S(T)+I(T)$. $X(T)$ is the population density of the middle trophic level species (intermediate predator) at time $T$ and $Y(T)$ is the population density of highest trophic level species (top predator) at time $T$.

$$
\begin{aligned}
& \frac{d S}{d T}=S\left[h_{1}-h_{2}(S+I)-h_{3} I-h_{4} X\right]+h_{5} I \\
& \frac{d I}{d T}=I\left(h_{3} S-h_{6} X-h_{5}-h_{7}\right) \\
& \frac{d X}{d T}=X\left(h_{8}-h_{9} X+h_{10} S+h_{11} I-h_{12} Y\right) \\
& \frac{d Y}{d T}=Y\left[h_{13}-h_{14} Y+h_{15} X-q E\right]
\end{aligned}
$$


It is assumed that, all the model parameters are positive values. The prey $N(T)$ grows with intrinsic growth rate $h_{1}$ and carrying capacity $h_{1} h_{2}^{-1}$ in absence of predation. The intermediate predator grows logistically with intrinsic growth rate $h_{8}$ and carrying capacity $h_{8} h_{9}^{-1}$, also the top predator grows logistically with intrinsic growth rate $h_{13}$ and carrying capacity $h_{13} h_{14}^{-1}$. The predator $X$ consumes the prey $S$ and $I$ with maximum attack rates $h_{4}$ and $h_{6}$ respectively, while predator $Y$ preys upon $X$ according to maximum attack rate $h_{12}$. The parameters $h_{10}, h_{11}$ and $h_{15}$ are conversion rates of prey to predator for species $X$ and $Y$ respectively, for which $h_{4}>h_{10}, h_{6}>h_{11}$ and $h_{12}>h_{15}$. The parameter $h_{7}$ is disease induced mortality rate for species $I$. Finally, $q>0$ is the catch ability co-efficient of the predator, $E>0$ is the harvesting effort and $q E Y$ is the catch-rate function based on the CPUE (catch-per-uniteffort) hypothesis.

The Jacobian matrix of system (1) is $J=\left(\beta_{i j}\right) \in \mathfrak{R}^{4 \times 4}$, with entries

$\beta_{11}=h_{1}-2 h_{2} S-h_{2} I-h_{3} I-h_{4} X ; \beta_{13}=-h_{4} S ;$

$\beta_{12}=h_{5}-h_{2} S-h_{3} S ; \beta_{14}=0 ; \beta_{21}=h_{3} I ;$

$\beta_{22}=h_{3} S-h_{6} X-h_{5}-h_{7} ; \beta_{23}=-h_{6} I$;

$\beta_{24}=0 ; \beta_{31}=h_{10} X ; \beta_{32}=h_{11} X ; \beta_{34}=-h_{12} X$;

$\beta_{33}=h_{8}-2 h_{9} X+h_{10} S+h_{11} I-h_{12} Y$;

$\beta_{41}=\beta_{42}=0 ; \beta_{43}=h_{15} Y$;

$\beta_{44}=h_{13}-2 h_{14} Y+h_{15} X-q E$

By introducing the total environment population $\mathrm{P}(T)=S(T)+I(T)+X(T)+Y(T)$, summing the equation (1) and bounding the right-hand from above, following the steps of [27], boundedness of the solution trajectories of this model is established. In particular,

$\lim _{T \rightarrow \infty}(S(T)+I(T)) \leq \frac{h_{1}}{h_{2}} ; \quad \lim _{T \rightarrow \infty} X(T) \leq \frac{h_{8}}{h_{9}} ;$

$\lim _{T \rightarrow \infty} Y(T) \leq \frac{h_{13}}{h_{14}}$ and $\lim _{T \rightarrow \infty} P(T) \leq \varphi\left(\frac{h_{1} h_{9}+h_{2} h_{8}}{h_{2} h_{9}}\right)$

where $0<\varphi<\min \left\{h_{7}, q E\right\}$

In what follows, the system's equilibria are $E_{k}$ and we denote by $J_{k}$ and $\beta_{i j}^{[k]}$ the Jacobian and its entries evaluated at $E_{k}, i=1,2,3,4$, $j=1,2,3,4, k=0,1, \ldots, 11$.

\section{Analysis of System}

Clearly the origin equilibrium point $E_{0}=(0,0,0,0)$ is a trivial solution of the system (1), and the Jacobian matrix at $E_{0}$ becomes a triangular matrix, then the eigenvalues are $\quad \lambda_{S}^{[0]}=h_{1}>0, \lambda_{X}^{[0]}=h_{8}>0$ $\lambda_{I}^{[0]}=-\left(h_{5}+h_{7}\right)<0$ and $\lambda_{Y}^{[0]}=h_{13}-q E$. Yield, $E_{0}$ is a saddle point (unstable). By the same way, the Jacobian matrix at the equilibrium point $E_{1}=\left(S_{1}, 0,0,0\right) \quad$ where $\quad S_{1}=h_{1} h_{2}^{-1}$ becomes a triangular matrix, and the eigenvalues are

$$
\begin{aligned}
& \lambda_{S}^{[1]}=-h_{1}<0, \lambda_{I}^{[1]}=h_{3} S_{1}-\left(h_{5}+h_{7}\right), \\
& \lambda_{X}^{[1]}=h_{8}+h_{10} S_{1}>0 \text { and } \lambda_{Y}^{[1]}=h_{13}-q E .
\end{aligned}
$$

Therefore the equilibrium point $E_{1}$ is always saddle point (unstable). The equilibrium point $E_{2}=\left(0,0, X_{2}, 0\right)$ where $x_{2}=h_{8} h_{9}^{-1}$ has the following characteristic equation:

$$
\begin{aligned}
P\left(\lambda^{[2]}\right) & =\left(\left(h_{1}-h_{4} X_{2}\right)-\lambda_{S}^{[2]}\right)\left(-h_{8}-\lambda_{X}^{[2]}\right) \\
& \times\left(\left(h_{13}+h_{15} X_{2}-q E\right)-\lambda_{Y}^{[2]}\right) \\
& \times\left(-\left(h_{6} X_{2}+h_{5}+h_{7}\right)-\lambda_{I}^{[2]}\right)=0
\end{aligned}
$$

So, the eigenvalues are $\lambda_{I}^{[2]}=-\left(h_{6} X_{2}+h_{5}+h_{7}\right)<0$

$\lambda_{S}^{[2]}=h_{1}-h_{4} X_{2}, \lambda_{X}^{[2]}=-h_{8}<0$ and $\lambda_{Y}^{[2]}=h_{13}+h_{15} X_{2}-q E$.

Therefore the equilibrium point $E_{2}$ is locally asymptotically stable if and only if $h_{1} h_{4}^{-1}<X_{2}<\left(q E-h_{13}\right) h_{15}^{-1}$ and $h_{13}<q E$ hold. Otherwise, $E_{2}$ is saddle point (unstable). The equilibrium point is $E_{3}=\left(0,0,0, Y_{3}\right)$ where $Y_{3}=\left(h_{13}-q E\right) h_{14}^{-1}$ and the necessary and sufficient condition for existence $E_{3}$ is $h_{13}>q E$, the eigenvalues are

$\lambda_{X}^{[3]}=h_{8}-h_{12} Y_{3}$

$\lambda_{S}^{[3]}=h_{1}>0, \lambda_{I}^{[3]}=-\left(h_{5}+h_{7}\right)<0$, and

$\lambda_{Y}^{[3]}=-\left(h_{13}-q E\right)<0$. 
Therefore the equilibrium point $E_{3}$ is saddle point (unstable). The equilibrium point $E_{4}=\left(S_{4}, I_{4}, 0,0\right)$ where $S_{4}=\left(h_{5}+h_{7}\right) h_{3}^{-1}$ and $I_{4}=\frac{S_{4}\left(h_{1}-h_{2} S_{4}\right)}{\left(h_{2} S_{4}+h_{7}\right)}$, the necessary and sufficient condition for existence $E_{4}$ is $h_{1} h_{2}^{-1}>S_{4}$, and the characteristic equation is:

$$
\begin{aligned}
& P\left(\lambda^{[4]}\right)=\left(\left(h_{8}+h_{10} S_{4}+h_{11} I_{4}\right)-\lambda_{X}^{[4]}\right)\left(\left(h_{13}-q E\right)-\lambda_{Y}^{[4]}\right) \\
& \times\left(\begin{array}{c}
\lambda^{2}-\left(h_{1}-2 h_{2} S_{4}-\left(h_{2}+h_{3}\right) I_{4}\right) \lambda \\
+h_{3} I_{4}\left(h_{2} S_{4}+h_{7}\right)
\end{array}\right)=0
\end{aligned}
$$

So, the eigenvalues are $\lambda_{X}^{[4]}=h_{8}+h_{10} S_{4}+h_{11} I_{4}>0, \lambda_{Y}^{[4]}=h_{13}-q E$ and $\lambda_{S}^{[4]} \times \lambda_{I}^{[4]}=h_{3} I_{4}\left(h_{2} S_{4}+h_{7}\right)>0$ $\lambda_{S}^{[4]}+\lambda_{I}^{[4]}=h_{1}-2 h_{2} S_{4}-h_{2} I_{4}-h_{3} I_{4}$.

Therefore the equilibrium point $E_{4}$ is saddle point (unstable). Now, we turn to the investigation of equilibria $E_{k}=\left(S_{k}, I_{k}, X_{k}, Y_{k}\right), k=5,6, \ldots, 11$ of system (1). The equilibrium points in which simple prey-predator model namely $E_{5}=\left(S_{5}, 0, X_{5}, 0\right)$ and $E_{7}=\left(0,0, X_{7}, Y_{7}\right)$ where

$S_{5}=\frac{\left(h_{1} h_{9}-h_{4} h_{8}\right)}{\left(h_{4} h_{10}+h_{2} h_{9}\right)}, \quad X_{5}=\frac{\left(h_{1} h_{10}+h_{2} h_{8}\right)}{\left(h_{4} h_{10}+h_{2} h_{9}\right)}$ with the feasibility conditions $h_{4} h_{8}<h_{1} h_{9}$, and $X_{7}=\frac{\left(h_{8} h_{14}+h_{12} q E-h_{12} h_{13}\right)}{\left(h_{9} h_{14}-h_{12} h_{15}\right)}$,

$Y_{7}=\frac{\left(h_{8}-h_{9} X_{7}\right)}{h_{12}}$ with feasibility conditions $h_{8} h_{14}+h_{12} q E>h_{12} h_{13}$ and $X_{7}<h_{8} h_{9}^{-1}$

respectively. The equilibrium point $E_{6}=\left(S_{6}, 0,0, Y_{6}\right) \quad$ where $S_{6}=h_{1} h_{2}^{-1} \quad$ and $Y_{6}=\left(h_{13}-q E\right) h_{14}^{-1}$ with the feasibility condition $h_{13}>q E$. Also, we have EcoEpidemiological model with equilibrium point $E_{8}=\left(S_{8}, I_{8}, X_{8}, 0\right)$ where

$X_{8}=\frac{\left(h_{3} S_{8}-h_{5}-h_{7}\right)}{h_{6}}$,

$I_{8}=\frac{\left(h_{3} h_{9}-h_{6} h_{10}\right) S_{8}-\left(h_{6} h_{8}+h_{9}\left(h_{5}+h_{7}\right)\right)}{h_{6} h_{11}}$

while $S_{8}$ represents a positive root of the equation $A_{1} S^{2}+A_{2} S+A_{3}=0$ where

$$
\begin{aligned}
& A_{1}=-\left[\begin{array}{c}
h_{2} h_{6} h_{11}+\left(h_{2}+h_{3}\right)\left(h_{3} h_{9}-h_{6} h_{10}\right) \\
+h_{3} h_{4} h_{11}
\end{array}\right]<0 \\
& A_{2}=h_{1} h_{6} h_{11}+\left(h_{2}+h_{3}\right)\left[h_{6} h_{8}+h_{9}\left(h_{5}+h_{7}\right)\right] \\
& +h_{4} h_{11}\left(h_{5}+h_{7}\right)+h_{5}\left(h_{3} h_{9}-h_{6} h_{10}\right)>0 \\
& A_{3}=-h_{5}\left[h_{6} h_{8}+h_{9}\left(h_{5}+h_{7}\right)\right]<0
\end{aligned}
$$

Obviously, $E_{8}$ exists uniquely in the interior of the first octant of SIX-space if and only if $h_{3} h_{9}>h_{6} h_{10}$ and $S_{8}>\max \left\{\frac{\left(h_{5}+h_{7}\right)}{h_{3}}, \frac{h_{6} h_{8}+h_{9}\left(h_{5}+h_{7}\right)}{\left(h_{3} h_{9}-h_{6} h_{10}\right)}\right\}$.

The equilibrium point $E_{9}=\left(S_{9}, I_{9}, 0, Y_{9}\right)$ where components

$S_{9}=\frac{\left(h_{5}+h_{7}\right)}{h_{3}}, I_{9}=\frac{\left(h_{1}-h_{2} S_{9}\right) S_{9}}{\left(h_{7}+h_{2} S_{9}\right)}$ and $Y_{9}=\frac{\left(h_{13}-q E\right)}{h_{14}}$ with feasibility conditions $S_{9}<\frac{h_{1}}{h_{2}}$ and $h_{13}>q E$. We have food chain model with equilibrium point

$E_{10}=\left(S_{10}, 0, X_{10}, Y_{10}\right)$ where:

$S_{10}=\frac{\left[\begin{array}{c}h_{1} h_{9} h_{14}+h_{4} h_{12} h_{13}+h_{1} h_{12} h_{15} \\ -h_{4} h_{8} h_{14}-h_{4} h_{12} q E\end{array}\right]}{\left[h_{2} h_{9} h_{14}+h_{4} h_{10} h_{14}+h_{2} h_{12} h_{15}\right]}$,

$X_{10}=\frac{\left(h_{1}-h_{2} S_{10}\right)}{h_{4}}, Y_{10}=\frac{\left(h_{13}+h_{15} X_{10}-q E\right)}{h_{14}}$

with feasibility conditions

$h_{4} h_{8} h_{14}+h_{4} h_{12} q E<h_{1} h_{9} h_{14}+h_{4} h_{12} h_{13}+h_{1} h_{12} h_{15}$ ， $S_{10}<h_{1} h_{2}^{-1}$

and $h_{13}+h_{15} X_{10}>q E$. Finally, we have the coexistence equilibrium point $E_{11}=\left(S_{11}, I_{11}, X_{11}, Y_{11}\right)$ with components

$S_{11}=\frac{\left(h_{6} X_{11}+h_{5}+h_{7}\right)}{h_{3}}, Y_{11}=\frac{\left(h_{15} X_{11}+h_{13}-q E\right)}{h_{14}}$

$I_{11}=\frac{S_{11}\left(h_{1}-h_{2} S_{11}-h_{4} X_{11}\right)}{\left(h_{2} S_{11}+h_{6} X_{11}+h_{7}\right)}$,

while $X_{11}$ represents a positive root of the following second order polynomial equation $A_{1} X^{2}+A_{2} X+A_{3}=0$ where 


$$
\begin{aligned}
A_{1}= & h_{6}\left[h_{6} h_{10} h_{14}-h_{3} h_{9} h_{14}-h_{12} h_{15}\right]\left(h_{2}-h_{3}\right) \\
& +h_{6} h_{11} h_{14}\left(h_{2} h_{6}-h_{3} h_{4}\right) \\
A_{2} & =\left(h_{2} h_{5}+h_{2} h_{7}+h_{3} h_{7}\right)\left[h_{6} h_{10} h_{14}-h_{3} h_{9} h_{14}-h_{12} h_{15}\right] \\
& +h_{6} h_{11} h_{14}\left[h_{1} h_{3}-2 h_{2}\left(h_{5}+h_{7}\right)\right] \\
& +\left[h_{5} h_{6} h_{10} h_{14}+h_{6} h_{7} h_{10} h_{14}-h_{6} h_{12} h_{13}+h_{6} h_{12} q E\right] \\
& \times\left(h_{2}-h_{3}\right)-h_{3} h_{4} h_{11} h_{14}\left(h_{5}+h_{7}\right) \\
A_{3} & =\left[h_{3} h_{8} h_{14}+h_{5} h_{10} h_{14}+h_{7} h_{10} h_{14}-h_{12} h_{13}+h_{12} q E\right] \\
& \times\left(h_{2} h_{5}+h_{2} h_{7}+h_{3} h_{7}\right)+h_{3} h_{6} h_{8} h_{14}\left(h_{2}-h_{3}\right) \\
& +\left(h_{5}+h_{7}\right)\left[h_{1} h_{3}-h_{2}\left(h_{5}+h_{7}\right)\right]
\end{aligned}
$$

Therefore, straight forward computation shows that $E_{11}$ exists uniquely in the int. $\mathfrak{R}_{+}^{4}$ if and only if $h_{13}+h_{15} X_{11}>q E$, $h_{1}>h_{2} S_{11}+h_{4} X_{11}$ and one set the following conditions holds

$$
\begin{aligned}
& A_{1}>0 \text { and } A_{3}<0 \\
& \text { or } \\
& A_{1}<0 \text { and } A_{3}>0
\end{aligned}
$$

\section{Theorem (1):}

The equilibrium point $E_{5}$ is locally asymptotically stable in $\mathfrak{R}_{+}^{4}$ if and only if:

$$
\begin{aligned}
& h_{13}+h_{15} X_{5}<q E, h_{3} S_{5}<h_{6} X_{5}+h_{5}+h_{7} \\
& 2 h_{8}<\min \left\{h_{2} S_{5}+3 h_{9} X_{5}, \frac{\left[h_{4} h_{10}+3 h_{2} h_{9}\right] x_{5}}{h_{2}}\right\} \ldots . .
\end{aligned}
$$

\section{Proof:}

The Jacobian matrix of the system (1) at $E_{5}$ is given by:

$$
J_{5}=\left(\begin{array}{cccc}
-h_{2} S_{5} & \left(\begin{array}{c}
h_{5}-h_{2} S_{5} \\
-h_{3} S_{5}
\end{array}\right) & -h_{4} S_{5} & 0 \\
0 & \left(\begin{array}{c}
h_{3} S_{5}-h_{6} X_{5} \\
-h_{5}-h_{7}
\end{array}\right) & 0 & 0 \\
h_{10} X_{5} & h_{11} X_{5} & \left(2 h_{8}-3 h_{9} X_{5}\right) & -h_{12} X_{5} \\
0 & 0 & 0 & \left(\begin{array}{c}
h_{15} X_{5} \\
h_{13}-q E
\end{array}\right)
\end{array}\right)
$$

So, the characteristic equation of $J_{5}$ can be written by

$$
\begin{aligned}
P\left(\lambda^{[5]}\right) & =\left(\begin{array}{l}
\left(\lambda^{[5]}\right)^{2}+\left(h_{2} S_{5}-2 h_{8}+3 h_{9} X_{5}\right) \lambda^{[5]} \\
+S_{5}\left(h_{4} h_{10} X_{5}-h_{2}\left(2 h_{8}-3 h_{9} X_{5}\right)\right)
\end{array}\right) \\
& \times\left(\left(h_{3} S_{5}-h_{6} X_{5}-h_{5}-h_{7}\right)-\lambda_{I}^{[5]}\right) \\
& \times\left(\left(h_{13}+h_{15} X_{5}-q E\right)-\lambda_{Y}^{[5]}\right)
\end{aligned}
$$

from which, we obtain that:

$\lambda_{I}^{[5]}=h_{3} S_{5}-h_{6} X_{5}-h_{5}-h_{7}, \lambda_{Y}^{[5]}=h_{13}+h_{15} X_{5}-q E$ $\lambda_{S}^{[5]}+\lambda_{X}^{[5]}=-\left(h_{2} S_{5}-2 h_{8}+3 h_{9} X_{5}\right)$, $\lambda_{S}^{[5]} \times \lambda_{X}^{[5]}=S_{5}\left[h_{4} h_{10} X_{5}-h_{2}\left(2 h_{8}-3 h_{9} x_{5}\right)\right]$
Here $\lambda_{S}^{[5]}, \lambda_{I}^{[5]}, \lambda_{X}^{[5]}$ and $\lambda_{Y}^{[5]}$ denote to the eigenvalues in the $S$-direction, $I$-direction, $X$-direction and $Y$-direction, respectively. So, it is easy to verify that, all the eigenvalues have negative real parts if and only if the condition (2) holds. Therefore, the equilibrium point $E_{5}$ is locally asymptotically stable in $\mathfrak{R}_{+}^{4}$.

\section{Theorem (2):}

The equilibrium point $E_{6}$ is locally asymptotically stable in $\Re_{+}^{4}$ if and only if:

$h_{8}+h_{10} S_{6}<h_{12} Y_{6}$
$h_{3} S_{6}<\left(h_{5}+h_{7}\right)$

\section{Proof:}

The Jacobian matrix of the system (1) at $E_{6}$ is given by:

$$
J_{6}=\left(\begin{array}{cccc}
-h_{1}\left(\begin{array}{c}
h_{5}-h_{2} S_{6} \\
-h_{3} S_{6}
\end{array}\right) & -h_{4} S_{6} & 0 \\
0 & \left(\begin{array}{c}
h_{3} S_{6} \\
-h_{5}-h_{7}
\end{array}\right) & 0 & 0 \\
0 & 0 & \left(\begin{array}{c}
h_{8}+h_{10} S_{6} \\
-h_{12} Y_{6} \\
h_{15} Y_{6}
\end{array}\right. & -\left(h_{13}-q E\right)
\end{array}\right)
$$

So, the characteristic equation of $J_{6}$ can be written by

$$
\begin{aligned}
& P\left(\lambda^{[6]}\right)=\left(\left(h_{3} S_{6}-h_{5}-h_{7}\right)-\lambda_{I}^{[6]}\right)\left(-\left(h_{13}-q E\right)-\lambda_{\Upsilon}^{[6]}\right) \\
& \times\left(\left(h_{8}+h_{10} S_{6}-h_{12} Y_{6}\right)-\lambda_{X}^{[6]}\right)\left(-h_{1}-\lambda_{S}^{[6]}\right)
\end{aligned}
$$

from which, we obtain that:

$$
\begin{aligned}
& \lambda_{S}^{[6]}=-h_{1}<0, \lambda_{I}^{[6]}=\left(h_{3} S_{6}-h_{5}-h_{7}\right), \\
& \lambda_{X}^{[6]}=\left(h_{8}+h_{10} S_{6}-h_{12} Y_{6}\right), \lambda_{Y}^{[5]}=-\left(h_{13}-q E\right)<0
\end{aligned}
$$

Here $\lambda_{S}^{[6]}, \lambda_{I}^{[6]}, \lambda_{X}^{[6]}$ and $\lambda_{Y}^{[6]}$ denote to the eigenvalues in the $S$-direction, $I$-direction, $X$-direction and $Y$-direction, respectively. So, it is easy to verify that, all the eigenvalues have negative real parts if and only if the condition (3) holds. Therefore, the equilibrium point $E_{6}$ is locally asymptotically stable in $\mathfrak{R}_{+}^{4}$.

\section{Theorem (3):}

The equilibrium point $E_{7}$ is locally asymptotically stable in $\Re_{+}^{4}$ if and only if $h_{1} h_{4}^{-1}<X_{7}$. 
Proof:

The Jacobian matrix of the system (1) at $E_{7}$ is given by:

$J_{7}=\left(\begin{array}{cccc}\left(\begin{array}{c}h_{1} \\ -h_{4} X_{7}\end{array}\right) & h_{5} & 0 & 0 \\ 0 & -\left(\begin{array}{c}h_{6} X_{7} \\ +h_{5}+h_{7}\end{array}\right) & 0 & 0 \\ h_{10} X_{7} & h_{11} X_{7} & -h_{9} X_{7} & -h_{12} X_{7} \\ 0 & 0 & h_{15} Y_{7} & -h_{14} Y_{7}\end{array}\right)$

So, the characteristic equation of $J_{7}$ can be written by

$$
\begin{aligned}
& P\left(\lambda^{[7]}\right)=\left(\left(h_{1}-h_{4} X_{7}\right)-\lambda_{S}^{[7]}\right)\left(-\left(h_{6} X_{7}+h_{5}+h_{7}\right)-\lambda_{I}^{[7]}\right) \\
& \quad \times\left(\begin{array}{l}
\left(\lambda^{[7]}\right)^{2}+\left(h_{9} X_{7}+h_{14} Y_{7}\right) \lambda^{[7]} \\
+\left(h_{9} h_{14}+h_{12} h_{15}\right) X_{7} Y_{7}
\end{array}\right)
\end{aligned}
$$

from which, we obtain that

$$
\begin{aligned}
& \lambda_{S}^{[7]}=h_{1}-h_{4} X_{7}, \lambda_{I}^{[7]}=-\left(h_{6} X_{7}+h_{5}+h_{7}\right)<0 \\
& \lambda_{X}^{[7]}+\lambda_{Y}^{[7]}=-\left(h_{9} X_{7}+h_{14} Y_{7}\right)<0, \\
& \lambda_{X}^{[7]} \times \lambda_{Y}^{[7]}=h_{9} h_{14}+h_{12} h_{15}>0
\end{aligned}
$$

Here $\lambda_{S}^{[7]}, \lambda_{I}^{[7]}, \lambda_{X}^{[7]}$ and $\lambda_{Y}^{[7]}$ denote to the eigenvalues in the $S$-direction, $I$-direction, $X$-direction and $Y$-direction, respectively. So, it is easy to verify that, all the eigenvalues have negative real parts if and only if $h_{1} h_{4}^{-1}<X_{7}$ holds. Therefore, the equilibrium point $E_{7}$ is locally asymptotically stable in $\mathfrak{R}_{+}^{4}$.

\section{Theorem (4):}

If the following conditions hold

$$
\left.\begin{array}{l}
h_{13}+h_{15} X_{8}<q E \\
h_{1}<2 h_{2} S_{8}+h_{3} I_{8}+h_{4} X_{8} \\
h_{3} h_{4} S_{8}<h_{6} h_{9} X_{8}
\end{array}\right\}
$$

Then, the equilibrium point $E_{8}$ is a locally asymptotically stable.

\section{Proof:}

The Jacobian matrix of the system (1) at $E_{8}$ is given by:

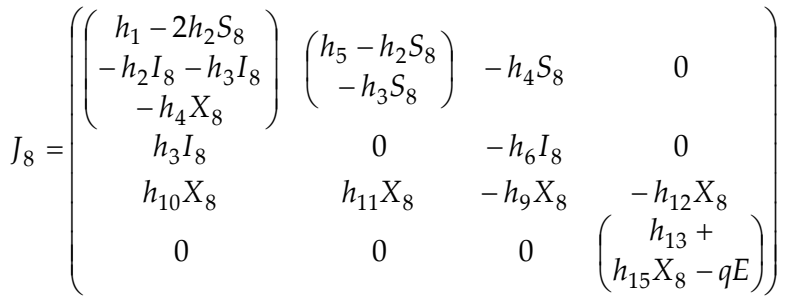

So, the characteristic equation of $J_{8}$ can be written by

$$
\begin{aligned}
P\left(\lambda^{[8]}\right)=\left(\lambda_{Y}^{[8]}-\left(h_{13}+h_{15} X_{8}-q E\right)\right) & \\
\times & {\left[\left(\lambda^{[8]}\right)^{3}+F_{1}\left(\lambda^{[8]}\right)^{2}+F_{2}\left(\lambda^{[8]}\right)+F_{3}\right] \text { with } } \\
F_{1}= & -h_{1}+2 h_{2} S_{8}+\left(h_{2}+h_{3}\right) I_{8}+\left(h_{4}+h_{9}\right) X_{8} \\
F_{2}= & h_{3} I_{8}\left(h_{2} S_{8}+h_{3} S_{8}-h_{5}\right)+h_{2} h_{9} I_{8} \\
& -h_{9} X_{8}\left(h_{1}-2 h_{2} S_{8}-h_{3} I_{8}-h_{4} X_{8}\right) \\
& +h_{4} h_{10} S_{8} X_{8}+h_{6} h_{11} I_{8} X_{8}
\end{aligned}
$$

Here $\lambda_{Y}^{[8]}$ denote to the eigenvalue in the $Y$-direction. The Routh-Hurwitz conditions require $F_{i}>0 \forall i=1,3$ and $\Delta=F_{1} F_{2}-F_{3}>0$, follows from condition (4b) and in addition the negativity of the other eigenvalues, namely condition (4a). So, according to RouthHurwitz criterion $E_{8}$ is locally asymptotically stable.

\section{Theorem (5):}

The equilibrium point $E_{9}$ is locally asymptotically stable in $\mathfrak{R}_{+}^{4}$ if and only if $\left(h_{8}+h_{10} S_{9}+h_{11} I_{9}\right) h_{12}^{-1}<Y_{9}$

\section{Proof:}

The Jacobian matrix of the system (1) at $E_{9}$ is given by:

$J_{9}=\left(\begin{array}{cccc}\beta_{11}^{[9]} & \beta_{12}^{[9]} & \beta_{13}^{[9]} & 0 \\ \beta_{21}^{[9]} & 0 & \beta_{23}^{[9]} & 0 \\ 0 & 0 & \beta_{33}^{[9]} & 0 \\ 0 & 0 & \beta_{43}^{[9]} & \beta_{44}^{[9]}\end{array}\right)$

where:

$$
\begin{aligned}
& \beta_{11}^{[9]}=h_{1}-2 h_{2} S_{9}-\left(h_{2}+h_{3}\right) I_{9} ; \beta_{12}^{[9]}=-\left(h_{2} S_{9}+h_{7}\right) \\
& \beta_{13}^{[9]}=-h_{4} S_{9} ; \beta_{21}^{[9]}=h_{3} I_{9} ; \beta_{23}^{[9]}=-h_{6} I_{9} ; \\
& \beta_{33}^{[9]}=h_{8}+h_{10} S_{9}+h_{11} I_{9}-h_{12} Y_{9} ; \\
& \beta_{43}^{[9]}=h_{15} Y_{9} ; \beta_{44}^{[9]}=-\left(h_{13}-q E\right)
\end{aligned}
$$

So, the characteristic equation of $J_{9}$ can be written by 


$$
\begin{aligned}
P\left(\lambda^{[9]}\right) & =\left(\left(h_{8}+h_{10} S_{9}+h_{11} I_{9}-h_{12} Y_{9}\right)-\lambda_{X}^{[9]}\right) \\
& \times\left(\begin{array}{c}
\left(\lambda^{[9]}\right)^{2}-\left(h_{1}-2 h_{2} S_{9}-\left(h_{2}+h_{3}\right) I_{9}\right) \lambda^{[9]} \\
+h_{3} I_{9}\left(h_{2} S_{9}+h_{7}\right)
\end{array}\right) \\
& \times\left(-\left(h_{13}-q E\right)-\lambda_{Y}^{[9]}\right)
\end{aligned}
$$

from which, we obtain that:

$$
\begin{aligned}
& \lambda_{X}^{[9]}=h_{8}+h_{10} S_{9}+h_{11} I_{9}-h_{12} Y_{9}, \\
& \lambda_{Y}^{[9]}=-\left(h_{13}-q E\right)<0 \\
& \lambda_{S}^{[9]}+\lambda_{I}^{[9]}=h_{1}-2 h_{2} S_{9}-\left(h_{2}+h_{3}\right) I_{9}, \\
& \lambda_{S}^{[9]} \times \lambda_{I}^{[9]}=h_{3} I_{9}\left(h_{2} S_{9}+h_{7}\right)>0
\end{aligned}
$$

Here $\lambda_{S}^{[9]}, \lambda_{I}^{[9]}, \lambda_{X}^{[9]}$ and $\lambda_{Y}^{[9]}$ denote to the eigenvalues in the $S$-direction, $I$-direction, $X$-direction and $Y$-direction, respectively. So, it is easy to verify that, all the eigenvalues have negative real parts if and only if the condition (5) holds. Therefore, the equilibrium point $E_{9}$ is locally asymptotically stable in $\Re_{+}^{4}$.

\section{Theorem (6):}

The equilibrium point $E_{10}$ is locally asymptotically stable in $\Re_{+}^{4}$ if and only if

$$
\begin{aligned}
& S_{10}<\left(h_{5}+h_{7}+h_{6} X_{10}\right) h_{3}^{-1} \ldots \ldots \\
& h_{8}+h_{10} S_{10}<2 h_{9} X_{10}+h_{12} Y_{10}
\end{aligned}
$$

\section{Proof:}

The Jacobian matrix of the system (1) at $E_{10}$ is given by:

$J_{10}=\left(\begin{array}{cccc}\beta_{11}^{[10]} & \beta_{12}^{[10]} & \beta_{13}^{[10]} & 0 \\ 0 & \beta_{22}^{[10]} & 0 & 0 \\ \beta_{31}^{[10]} & \beta_{32}^{[10]} & \beta_{33}^{[10]} & \beta_{34}^{[10]} \\ 0 & 0 & \beta_{43}^{[10]} & \beta_{44}^{[10]}\end{array}\right)$

where:

$\beta_{11}^{[10]}=-h_{2} S_{10} ; \beta_{12}^{[10]}=h_{5}-h_{2} S_{10}-h_{3} S_{10} ;$

$\beta_{13}^{[10]}=-h_{4} S_{10} ; \beta_{31}^{[10]}=h_{10} X_{10} ; \beta_{32}^{[10]}=h_{11} X_{10}$;

$\beta_{22}^{[10]}=h_{3} S_{10}-h_{6} X_{10}-h_{5}-h_{7} ; \beta_{34}^{[10]}=-h_{12} X_{10}$;

$\beta_{33}^{[10]}=h_{8}-2 h_{9} X_{10}+h_{10} S_{10}-h_{12} Y_{10}$;

$\beta_{43}^{[10]}=h_{15} Y_{10} ; \beta_{44}^{[10]}=-\left(h_{13}+h_{15} X_{10}-q E\right)$

So, the characteristic equation of $J_{10}$ can be written by

$$
\begin{aligned}
P\left(\lambda^{[10]}\right) & =\left(\lambda_{I}^{[10]}-\left(h_{3} S_{10}-h_{6} X_{10}-h_{5}-h_{7}\right)\right) \\
& \times\left[\left(\lambda^{[10]}\right)^{3}+F_{1}\left(\lambda^{[10]}\right)^{2}+F_{2}\left(\lambda^{[10]}\right)+F_{3}\right]
\end{aligned}
$$

with

$$
\begin{aligned}
& \begin{aligned}
F_{1}= & -\left(h_{8}-2 h_{9} X_{10}+h_{10} S_{10}-h_{12} Y_{10}\right) \\
& +h_{2} S_{10}+h_{14} Y_{10}
\end{aligned} \\
& \begin{aligned}
F_{2}= & h_{2} h_{14} S_{10} Y_{10}+X_{10}\left(h_{12} h_{15} Y_{10}+h_{4} h_{10} S_{10}\right) \\
& -\left(h_{8}-2 h_{9} X_{10}+h_{10} S_{10}-h_{12} Y_{10}\right) \\
& \times\left(h_{1} S_{10}+h_{14} Y_{10}\right)
\end{aligned} \\
& F_{3}=S_{10} Y_{10}\left[\begin{array}{l}
\left(h_{2} h_{12} h_{15}+h_{4} h_{10} h_{14}\right) X_{10}-h_{2} h_{14} \\
\times\left(h_{8}-2 h_{9} X_{10}+h_{10} S_{10}-h_{12} Y_{10}\right)
\end{array}\right] \\
& \begin{aligned}
\Delta= & F_{1} F_{2}-F_{3} \\
= & h_{4} h_{10} S_{10} X_{10}\left[h_{2} S_{10}-\left(\begin{array}{c}
h_{8}-2 h_{9} X_{10} \\
+h_{10} S_{10}-h_{12} Y_{10}
\end{array}\right)\right] \\
+ & h_{14} Y_{10}\left[h_{2} S_{10}-\left(h_{8}-2 h_{9} X_{10}+h_{10} S_{10}-h_{12} Y_{10}\right)\right] \\
\times & {\left[h_{2} S_{10}+h_{14} Y_{10}-\left(h_{8}-2 h_{9} X_{10}+h_{10} S_{10}-h_{12} Y_{10}\right)\right] }
\end{aligned} \\
& +h_{12} h_{15} X_{10} Y_{10}\left[h_{14} Y_{10}-\left(\begin{array}{c}
h_{8}-2 h_{9} X_{10} \\
+h_{10} S_{10}-h_{12} Y_{10}
\end{array}\right)\right]
\end{aligned}
$$

Here $\lambda_{I}^{[10]}$ denote to the eigenvalue in the $I$-direction. The Routh-Hurwitz conditions require $F_{i}>0 \forall i=1,3$ and $\Delta=F_{1} F_{2}-F_{3}>0$, follows from condition (6b) and in addition the negativity of the other eigenvalues, namely condition (6a). So, according to RouthHurwitz criterion $E_{10}$ is locally asymptotically stable.

\section{Theorem (7):}

If the following conditions hold

$h_{1}<2 h_{2} S_{11}+\left(h_{2}+h_{3}\right) I_{11}+h_{4} X_{11}$

$\frac{h_{6} h_{10}}{h_{9}}<h_{3}<\frac{h_{9} h_{10} X_{11}}{h_{11} I_{11}}$

$$
h_{9} h_{14} X_{11} Y_{11}>h_{3} I_{11}\left(h_{2} S_{11}+h_{11} X_{11}+h_{7}\right)
$$

Then, the equilibrium point $E_{11}$ is a locally asymptotically stable.

\section{Proof:}

The Jacobian matrix of the system (1) at $E_{11}$ is given by $J_{11}=\left(\beta_{i j}^{[11]}\right)$ where:

$\beta_{11}^{[11]}=h_{1}-2 h_{2} S_{11}-\left(h_{2}+h_{3}\right) I_{11}-h_{4} X_{11} ;$

$\beta_{12}^{[11]}=-\left(h_{7}+h_{2} S_{11}+h_{6} X_{11}\right) ; \beta_{13}^{[11]}=-h_{4} S_{11}$;

$\beta_{14}^{[11]}=0 ; \beta_{21}^{[11]}=h_{3} I_{11} ; \beta_{22}^{[11]}=0$ ，

$\beta_{23}^{[11]}=-h_{6} I_{11} ; \beta_{24}^{[11]}=0 ; \beta_{31}^{[11]}=h_{10} X_{11}$ ；

$\beta_{32}^{[11]}=h_{11} X_{11} ; \beta_{33}^{[11]}=-h_{9} X_{11} ; \beta_{34}^{[11]}=-h_{12} X_{11}$;

$\beta_{41}^{[11]}=\beta_{42}^{[11]}=0 ; \beta_{43}^{[11]}=h_{15} Y_{11} ; \beta_{44}^{[11]}=-h_{14} Y_{11}$.

So, the characteristic equation of $J_{11}$ can be written by

$$
\begin{aligned}
P\left(\lambda^{[11]}\right) & =\left(\lambda^{[11]}\right)^{4}+F_{1}\left(\lambda^{[11]}\right)^{3}+F_{2}\left(\lambda^{[11]}\right)^{2} \\
& +F_{3}\left(\lambda^{[11]}\right)+F_{4}
\end{aligned}
$$

with 


$$
\begin{aligned}
F_{1} & =-\left(\beta_{11]}^{[11]}+\beta_{33}^{[11]}+\beta_{44}^{[11]}\right) \\
F_{2} & =\beta_{11}^{[11]} \beta_{44}^{[11]}+\beta_{33}^{[11]} \beta_{44}^{[11]}-\beta_{12}^{[11]} \beta_{21}^{[11]}-\beta_{23}^{[11]} \beta_{32}^{[11]} \\
+ & \beta_{11}^{[11]} \beta_{33}^{[11]}-\beta_{13}^{[11]} \beta_{31}^{[11]}-\beta_{34}^{[11]} \beta_{43}^{[11]} \\
F_{3} & =\beta_{11}^{[11]} \beta_{34}^{[11]} \beta_{43}^{[11]}+\beta_{44}^{[11]} \beta_{12}^{[11]} \beta_{21}^{[11]}+\beta_{44}^{[11]} \beta_{23}^{[11]} \beta_{32}^{[11]} \\
& -\beta_{11]}^{[11]} \beta_{33}^{[11]} \beta_{44}^{[11]}-\beta_{12}^{[11]} \beta_{23}^{[11]} \beta_{31}^{[11]}-\beta_{13}^{[11]} \beta_{32}^{[11]} \beta_{21}^{[11]} \\
& +\beta_{44}^{[11]} \beta_{13}^{[11]} \beta_{31}^{[11]}+\beta_{11}^{[11]} \beta_{23}^{[11]} \beta_{32}^{[11]}+\beta_{33}^{[11]} \beta_{12}^{[11]} \beta_{21}^{[11]} \\
F_{4} & =\beta_{12}^{[11]} \beta_{21}^{[11]} \beta_{34}^{[11]} \beta_{43}^{[11]}+\beta_{12}^{[11]} \beta_{23}^{[11]} \beta_{31}^{[11]} \beta_{44}^{[11]} \\
& +\beta_{13}^{[11]} \beta_{32}^{[11]} \beta_{21}^{[11]} \beta_{44}^{[11]}-\beta_{11}^{[11]} \beta_{44}^{[11]} \beta_{23}^{[11]} \beta_{32}^{[11]} \\
& -\beta_{33}^{[11]} \beta_{44}^{[11]} \beta_{12}^{[11]} \beta_{21}^{[11]} \\
\Delta & =F_{1} F_{2}-F_{3}=\beta_{11}^{[11]} \beta_{12}^{[11]} \beta_{21}^{[11]}-\left(\beta_{11}^{[11]}\right)^{2} \beta_{44}^{[11]} \\
& -2 \beta_{11}^{[11]} \beta_{33}^{[11]} \beta_{44}^{[11]}-\left(\beta_{11}^{[11]}\right)^{2} \beta_{33}^{[11]}+\beta_{11}^{[11]} \beta_{13}^{[11]} \beta_{31}^{[11]} \\
& +\beta_{12}^{[11]} \beta_{23}^{[11]} \beta_{31}^{[11]}-\beta_{11}^{[11]}\left(\beta_{33}^{[11]}\right)^{2}-\left(\beta_{33}^{[11]}\right)^{2} \beta_{44}^{[11]} \\
& +\beta_{44}^{[11]} \beta_{34}^{[11]} \beta_{43}^{[11]}+\beta_{33}^{[11]} \beta_{23}^{[11]} \beta_{32}^{[11]}+\beta_{44}^{[11]} \beta_{34}^{[11]} \beta_{43}^{[11]} \\
& -\beta_{11]}^{[11]}\left(\beta_{44}^{[11]}\right)^{2}-\beta_{33}^{[11]}\left(\beta_{44}^{[11]}\right)^{2} \\
& +\beta_{13}^{[11]}\left(\beta_{32}^{[11]} \beta_{21}^{[11]}+\beta_{33}^{[11]} \beta_{31}^{[11]}\right) \\
\Delta & F_{3}-\left(F_{1}\right)^{2} F_{4}=\left(\beta_{11}^{[11]}\right)^{2} \beta_{34}^{[11]} \beta_{43}^{[11]} \\
& {\left[\beta_{13}^{[11]} \beta_{31}^{[11]}-\beta_{11}^{[11]} \beta_{44}^{[11]}-\beta_{11}^{[11]} \beta_{33}^{[11]}\right] } \\
& \left.+2 \beta_{33}^{[11]} \beta_{44}^{[11]}-\left(\beta_{33}^{[11]}\right)^{2}-\left(\beta_{44}^{[11]}\right)^{2}\right] \\
&
\end{aligned}
$$$$
-\left(\beta_{11}^{[11]}\right)^{2} \beta_{33}^{[11]} \beta_{44}^{[11]}\left[\begin{array}{l}
2 \beta_{13}^{[11]} \beta_{31}^{[11]}-\beta_{11}^{[11]} \beta_{44}^{[11]} \\
-\beta_{11]}^{[11]} \beta_{33}^{[11]}-2 \beta_{33}^{[11]} \beta_{44}^{[11]} \\
-\left(\beta_{33}^{[11]}\right)^{2}-\left(\beta_{44}^{[11]}\right)^{2} \\
+2 \beta_{12}^{[11]} \beta_{21}^{[11]}+2 \beta_{23}^{[11]} \beta_{32}^{[11]}
\end{array}\right]
$$$$
+\left(\beta_{11}^{[11]}\right)^{2}\left[\begin{array}{l}
\left(\beta_{44}^{[11]}\right)^{2} \beta_{13}^{[11]} \beta_{31}^{[11]}-\left(\beta_{44}^{[11]}\right)^{2} \beta_{12}^{[11]} \beta_{21}^{[11]} \\
-\left(\beta_{33}^{[11]}\right)^{2} \beta_{23}^{[11]} \beta_{32}^{[11]}+\beta_{33}^{[11]} \beta_{11}^{[1]} \beta_{32}^{[11]} \beta_{21}^{[11]} \\
+\beta_{13}^{[1]} \beta_{31}^{[11]} \beta_{23}^{[11]} \beta_{32}^{[11]}+\beta_{12}^{[11]} \beta_{21}^{[11]} \beta_{23}^{[11]} \beta_{32}^{[11]} \\
-\beta_{11}^{[11]} \beta_{33}^{[11]} \beta_{23}^{[11]} \beta_{32}^{[11]}
\end{array}\right]
$$$$
+\beta_{34}^{[11]} \beta_{43}^{[11]}\left[\begin{array}{l}
\beta_{11}^{[11]} \beta_{34}^{[11]} \beta_{43}^{[11]}\left(\beta_{33}^{[11]}+\beta_{44}^{[11]}\right) \\
+\beta_{44}^{[11]} \beta_{13}^{[11]} \beta_{31}^{[11]}\left(\beta_{33}^{[11]}+\beta_{44}^{[11]}\right) \\
+\beta_{44}^{[11]} \beta_{23}^{[11]} \beta_{32}^{[11]}\left(\beta_{33}^{[11]}+\beta_{44}^{[11]}\left(\beta_{32}^{[11]} \beta_{21}^{[11]}+\beta_{33}^{[11]} \beta_{31}^{[11]}\right)\right. \\
+\beta_{11}^{[11]} \beta_{23}^{[11]}\left(\beta_{33}^{[11]} \beta_{32}^{[11]}+\beta_{12}^{[11]} \beta_{31}^{[11]}\right) \\
+\beta_{11]}^{[11]} \beta_{23}^{[11]} \beta_{32}^{[11]}\left(\beta_{33}^{[11]}+\beta_{44}^{[11]}\right) \\
-\beta_{13}^{[11]} \beta_{32}^{[11]} \beta_{21}^{[11]}\left(\beta_{33}^{[11]}+\beta_{44}^{[11]}\right) \\
-\beta_{12}^{[11]} \beta_{23}^{[11]} \beta_{31}^{[11]}\left(\beta_{33}^{[11]}+\beta_{44}^{[11]}\right)
\end{array}\right]
$$$$
+\beta_{11}^{[11]} \beta_{33}^{[11]} \beta_{44}^{[11]}\left[\begin{array}{l}
\beta_{33}^{[11]} \beta_{44}^{[11]}\left(\beta_{33}^{[11]}+\beta_{33}^{[11]}\right) \\
-\beta_{44}^{[11]} \beta_{23}^{[11]} \beta_{32}^{[11]}-\beta_{33}^{[11]} \beta_{23}^{[11]} \beta_{32}^{[11]} \\
+\beta_{13}^{[11]} \beta_{32}^{[11]} \beta_{21}^{[11]}-\beta_{33}^{[11]} \beta_{23}^{[11]} \beta_{32}^{[11]} \\
-\beta_{44}^{[11]} \beta_{12}^{[11]} \beta_{21}^{[11]}-\beta_{12}^{[11]} \beta_{23}^{[11]} \beta_{31}^{[11]}
\end{array}\right]
$$$$
+\beta_{13}^{[11]} \beta_{32}^{[11]} \beta_{21}^{[11]}\left[\beta_{11}^{[11]}\left(\beta_{33}^{[11]}\right)^{2}-\beta_{11}^{[11]} \beta_{13}^{[11]} \beta_{31}^{[11]}\right.
$$$$
\beta_{13} \beta_{32} \beta_{21}\left[-\beta_{11}^{[11]} \beta_{12}^{[11]} \beta_{21}^{[11]}-\beta_{33}^{[11]} \beta_{23}^{[11]} \beta_{32}^{[11]}\right]
$$$$
+\left(\beta_{33}^{[11]} \beta_{31}^{[11]}+\beta_{32}^{[11]} \beta_{21}^{[11]}\left[\begin{array}{l}
\beta_{44}^{[11]} \beta_{31}^{[11]}\left(\beta_{13}^{[11]}\right)^{2} \\
-\beta_{32}^{[11]} \beta_{21}^{[11]}\left(\beta_{13}^{[11]}\right)^{2} \\
-\beta_{13}^{[11]}\left(\beta_{44}^{[11]}\right)^{3} \\
-\beta_{33}^{[11]} \beta_{13}^{[11]}\left(\beta_{44}^{[11]}\right)^{2} \\
+\beta_{44}^{[11]} \beta_{11}^{[11]} \beta_{21}^{[11]} \beta_{13}^{[11]} \\
-\beta_{11]}^{[11]} \beta_{33}^{[11]} \beta_{44}^{[11]} \beta_{13}^{[11]} \\
+\beta_{44}^{[11]} \beta_{23}^{[11]} \beta_{32}^{[11]} \beta_{13}^{[11]} \\
-2 \beta_{11}^{[11]} \beta_{33}^{[11]} \beta_{44}^{[11]} \beta_{13}^{[1]} \\
-\beta_{11}^{[11]} \beta_{13}^{[11]}\left(\beta_{44}^{[11]}\right)^{2}
\end{array}\right]\right.
$$$$
-\beta_{33}^{[11]} \beta_{44}^{[11]} \beta_{23}^{[11]} \beta_{32}^{[11]} \beta_{44}^{[11]} \beta_{12}^{[11]} \beta_{23}^{[11]} \beta_{31}^{[11]}
$$$$
-2 \beta_{11}^{[11]} \beta_{34}^{[11]} \beta_{43}^{[11]}\left(\beta_{33}^{[11]} \beta_{44}^{[11]}+\beta_{12}^{[11]} \beta_{21}^{[11]}\right)\left(\beta_{33}^{[11]}+\beta_{44}^{[11]}\right)
$$$$
+\beta_{23}^{[11]} \beta_{32}^{[11}\left[\left(\beta_{44}^{[11]}\right)^{2} \beta_{13}^{[11]} \beta_{31}^{[11]}+\beta_{11}^{[11]} \beta_{33}^{[11]} \beta_{23}^{[11]} \beta_{32}^{[11]}\right]
$$ 


$$
+\beta_{11}^{[11]} \beta_{44}^{[11]}\left[\begin{array}{l}
\left(\beta_{13}^{[11]} \beta_{31}^{[11]}+\beta_{12}^{[11]} \beta_{21}^{[11]}\right)^{2}+\beta_{13}^{[11]} \beta_{31}^{[11]} \\
\times \beta_{23}^{[11]} \beta_{32}^{[11]}+\left(\beta_{44}^{[11]}\right)^{2} \beta_{13}^{[11]} \beta_{31}^{[11]} \\
-\left(\beta_{44}^{[11]}\right)^{2} \beta_{12}^{[11]} \beta_{21}^{[11]}-\beta_{44}^{[11]} \beta_{12}^{[11]} \\
\times \beta_{23}^{[11]} \beta_{31}^{[11]}+\beta_{12}^{[11]} \beta_{21}^{[11]} \beta_{13}^{[11]} \beta_{31}^{[11]}
\end{array}\right]
$$$$
+\beta_{33}^{[11]} \beta_{44}^{[11]} \beta_{23}^{[11]} \beta_{32}^{[11]}\left(\begin{array}{l}
\beta_{13}^{[11]} \beta_{31}^{[11]}-\beta_{33}^{[11]} \beta_{44}^{[11]} \\
+\beta_{23}^{[11]} \beta_{32}^{[11]}-\left(\beta_{44}^{[11]}\right)^{2} \\
+\beta_{12}^{[11]} \beta_{21}^{[11]}
\end{array}\right)
$$$$
+\beta_{12}^{[11]} \beta_{23}^{[11]} \beta_{31}^{[11]}\left[\begin{array}{l}
\beta_{44}^{[11]}\left(\beta_{13}^{[11]} \beta_{31}^{[11]}+\beta_{12}^{[11]} \beta_{21}^{[11]}\right) \\
-\beta_{21}^{[11]} \beta_{13}^{[11]} \beta_{32}^{[11]}-\left(\beta_{44}^{[11]}\right)^{3} \\
+\beta_{23}^{[11]} \beta_{32}^{[11]}\left(\beta_{11}^{[11]}+\beta_{44}^{[11]}\right)
\end{array}\right]
$$

$$
+\left(\beta_{33}^{[11]} \beta_{21}^{[11]}-\beta_{23}^{[11]} \beta_{31}^{[11]}\right)\left[\begin{array}{l}
\beta_{11}^{[11]} \beta_{12}^{[11]} \beta_{21}^{[11]} \beta_{13}^{[11]} \\
+\beta_{11}^{[11]} \beta_{21}^{[11]}\left(\beta_{12}^{[11]}\right)^{2} \\
-\beta_{12}^{[11]} \beta_{33}^{[11]}\left(\beta_{11}^{[11]}\right)^{2} \\
-\beta_{11}^{[11]} \beta_{12}^{[11]}\left(\beta_{33}^{[11]}\right)^{2} \\
+\beta_{33}^{[11]} \beta_{12}^{[11]} \beta_{23}^{[11]} \beta_{32}^{[11]} \\
-\beta_{11}^{[11]} \beta_{33}^{[11]} \beta_{44}^{[11]} \beta_{12}^{[11]} \\
+\beta_{23}^{[11]} \beta_{31}^{[11]}\left(\beta_{12}^{[11]}\right)^{2} \\
+\beta_{12}^{[11]} \beta_{21}^{[11]} \beta_{13}^{[11]} \beta_{32}^{[11]} \\
+\beta_{33}^{[11]} \beta_{12}^{[11]} \beta_{13}^{[11]} \beta_{31}^{[11]}
\end{array}\right]
$$

So, if $\beta_{11}^{[11]}<0 ; \beta_{33}^{[11]} \beta_{21}^{[11]}<\beta_{23}^{[11]} \beta_{31}^{[11]}$ $\beta_{33}^{[11]} \beta_{31}^{[11]}<-\beta_{32}^{[11]} \beta_{21}^{[11]} ; \beta_{33}^{[11]} \beta_{44}^{[11]}>-\beta_{12}^{[11]} \beta_{21}^{[11]}$ and by using the Routh-Hurwitz conditions require $\quad F_{i}>0 \forall i=1,3,4, \Delta=F_{1} F_{2}-F_{3}>0$ and $\left(F_{1} F_{2}-F_{3}\right) F_{3}-\left(F_{1}\right)^{2} F_{4}>0$, which reduces to conditions (7a)-(7c). So, according to Routh-Hurwitz criterion $E_{11}$ is locally asymptotically stable.

\section{Numerical Simulations}

To study the system (1) numerically let's use the cont. line (-) for $x$, dash line (- -) for $y$, dot line(::) for $z$ and dash-dot line(-.-) for $p$ in the all of the following figures. Now, consider the following set of parameters

$$
\begin{aligned}
& h_{1}=0.8, h_{2}=0.02, h_{3}=0.7, h_{4}=0.7, h_{5}=0.3, \\
& h_{6}=0.6, h_{7}=0.18, h_{8}=0.9, h_{9}=0.03, h_{10}=0.6, \\
& h_{11}=0.5, h_{12}=0.3, h_{13}=0.7, h_{14}=0.07, \\
& h_{15}=0.2, q=0.6, E=0.5
\end{aligned}
$$

With initial point $(0.75,0.75,0.75,0.75)$. For this set of parameter (8), the solution trajectory of system (1) approaches to the equilibrium point

$$
E_{11}=(1.046,1.119,0.42,6.915) \text { see Fig.(1). }
$$

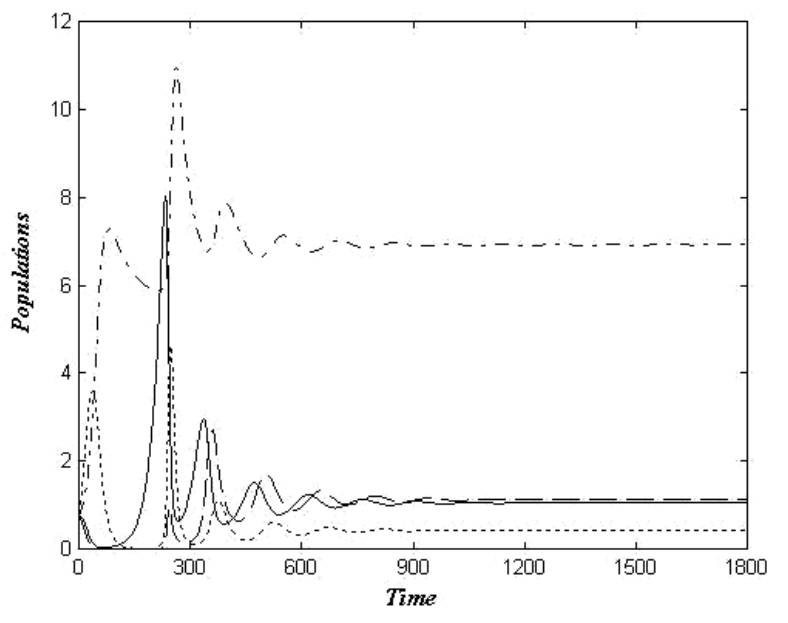

Fig.(1) Time series of the solution trajectory of system (1) for data given in Eq.(8) which show that $E_{11}$ is a locally asymptotically stable.

If the infection rates constant $h_{3} \leq 0.21$ then the solution trajectory of system (1) approaches to the equilibrium point $E_{10}=(2.923,0,1.059,8.741)$ see Fig.(2).

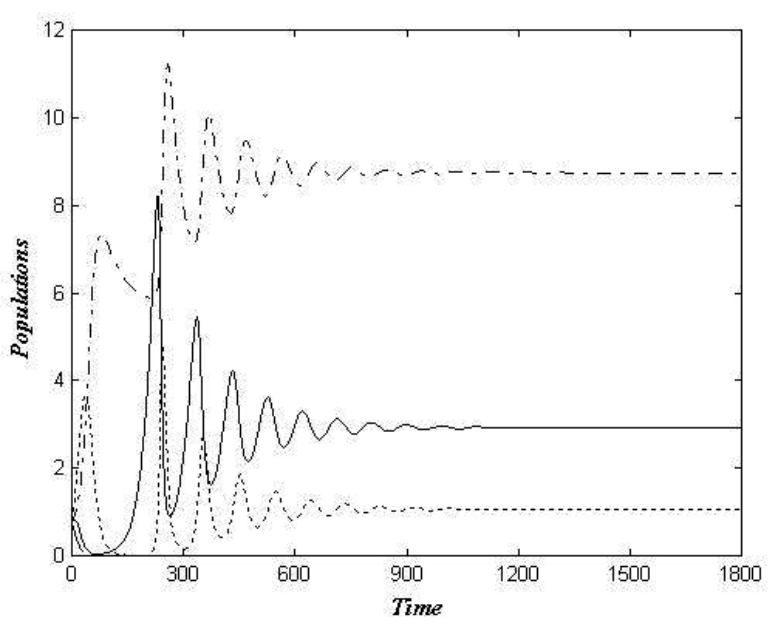

Fig.(2) Time series of the solution trajectory of system (1) for data given in Eq.(8) with

$$
h_{3}=0.21 \text {. }
$$

But, when the effect of catch-rate $q E$ is considered then the solution trajectory of system (1) approaches to the equilibrium point $E_{7}=(0,0,1.993,2.801)$ when $q \geq 0.95, E \geq 0.95$ see Fig.(3), and approaches to the equilibrium point $E_{9}=(0.686,2.783,0,9.714)$ when $q \leq 0.2, E \leq 0.1$ see Fig.(4). 


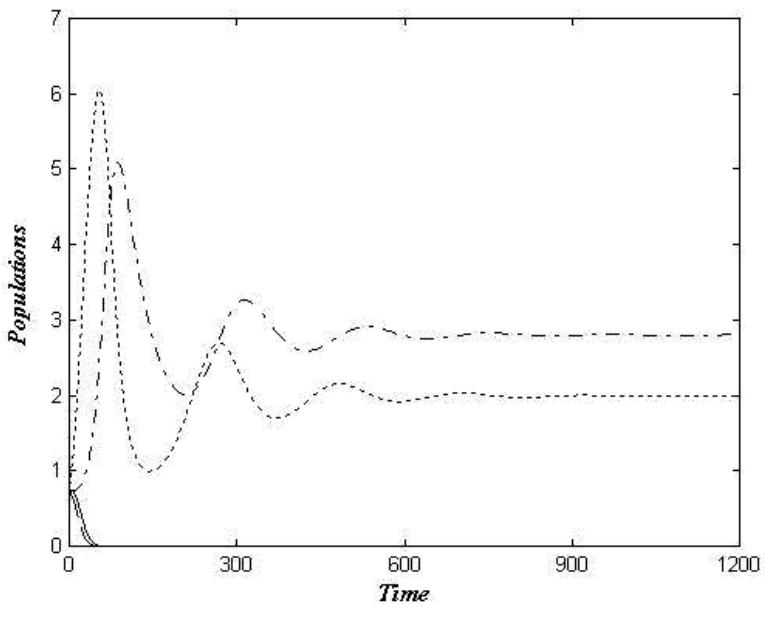

Fig.(3) Time series of the solution trajectory of system (1) for data given in Eq.(8) with $q=0.95, E=0.95$.

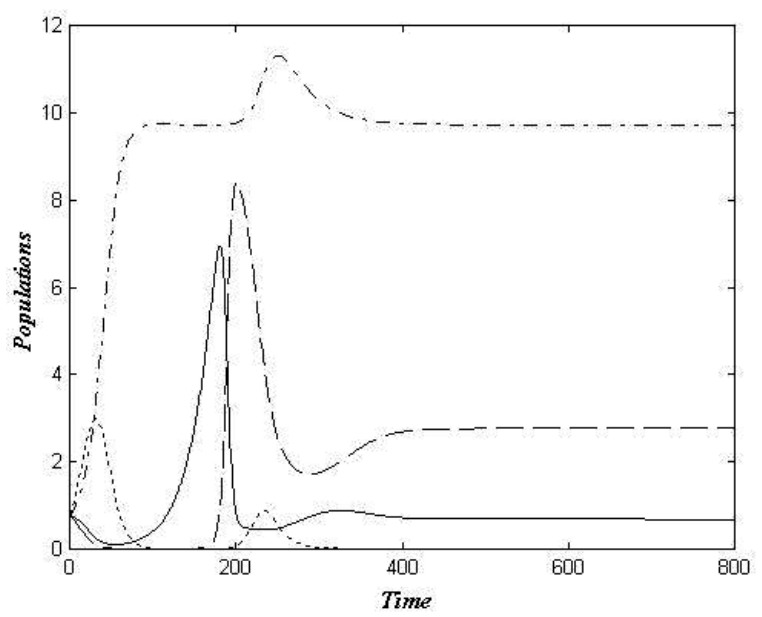

Fig.(4) Time series of the solution trajectory of system (1) for data given in Eq.(8) with $q=0.2, E=0.1$.

\section{Conclusions and Discussion}

In this paper, we proposed and analyzed the combined effect of SIS disease and harvest on a Food Chain model. The dynamical behaviour of system (1) has been investigated locally. In addition to assumed that the top predator population is harvested under optimal conditions, we used linear functional response and incidence rate for the diseases in prey species. The model included four non-linear autonomous differential equations that describe the dynamics of four different populations namely susceptible prey $(S)$, infected prey $(I)$, intermediate predator $(X)$ and top predator $(Y)$. The conditions for existence and stability for each equilibrium points are obtained. Similar, numerically explained that the solution trajectory of system (1) with parameters given in eq.(8) approaches to the equilibrium point $E_{11}=(0.891,1.602,0.24,7.428)$. The system (1) is solved numerically for varying of infection rate $h_{3}$ keeping other parameters fixed as given in Eq.(8), then the solutions trajectory of system (1) are drawn in Fig.(2), it is clear that, as the infection rate decreases the infected individuals started decreases and the system (1) return to asymptotically stable at the coexistence equilibrium point in the Int. $\mathfrak{R}_{+}^{4}$. However, decreasing $h_{3}$ further, say $h_{3}=0.21$, causes losing the stability and then the solutions trajectory approaches to another equilibrium point $E_{10}$. The same way with parameters $q \geq 0.95 ; E \geq 0.95$, but with $q \geq 0.2 ; E \geq 0.45$ the solutions trajectory approaches to another equilibrium point $E_{9}$.

\section{References}

[1] Gard T.C. and Hallam T.G., "Persistence in food webs-I. Lotka-Volterra food chains", Bull. Math. Biol., 41, 877-891, 1979.

[2] Gakkhar S. and Naji R.K., "Chaos in three species ratio dependent food chain", Chaos Solitons and Fractals 14, 771-778, 2002.

[3] Kesh D., Sarkar A.K. and Roy A.B., "Succession processes in a food web of a two autotrophy-one herbivore system", Biosystems 57, 129-138, 2000.

[4] Hsu S.B., Hwang T.W., and Kuang Y., "Rich dynamics of a ratio dependent oneprey two-predators model", J. Math. Biol., 43, 377-396, 2001.

[5] Raid Kamel Naji and Alla Tariq Balasim, "Chaos Dynamical behaviour of a three species food chain model with BeddingtonDeangelis functional response", Chaos, Solitons and Fractals 32, 1853-1866, 2007.

[6] Chasnov J. R., "Mathematical Biology", Jeffrey Robert Chasnov, USA, 2012.

[7] Andreasen V. and Sasaki A., "Shaping the phylogenetic tree of influenza by crossimmunity", Theoretical population biology, 70, 164-173, 2006.

[8] Brauer F., Wu J. and Driessche P., "Mathematical epidemiology", Berlin Heidelberg, Springer-Verlag, 2008.

[9] Diego J.R. and Lourdes T. S., "Models of infection diseases in spatially heterogeneous environments", Bulletin of Mathematical Biology, 63(3), 547-571, 2001. 
[10] Hethcote H. W., "The mathematics of infectious diseases", SIAM Review, 42(4), 599-653, 2000.

[11] Kapur J. N., "Mathematical models in biology \& medicine", New Delhi, Affiliated EWP Ltd, 1985.

[12] Kermack W. O. and McKendrick A. G., "Contributions to the mathematical theory of epidemics, Part 1", Proceeding of the Royal Society Series A, 115, 700-721, 1927.

[13] Murray J. D., "Mathematical biology an introduction, third edition", Berlin Heidelberg, Springer-Verlag, 2002.

[14] Arino O., Abdllaoui A., Mikram J., and Chattopadhyay J., "Infection in prey population may act as a biological control in ratio-dependent predator-prey models", Nonlinearity, 17, 1101-1116, 2004.

[15] Bakare E. A., Adekunle Y. and Nwagwo A., "Mathematical analysis of the control of the spread of infectious disease in a preypredator ecosystem", International Journal of Computer \& Organization Trends, 2(1), 27-32, 2012.

[16] Chattopadhyay J. and Arino O., "A predator-prey model with disease in the prey", Nonlinear Analysis, 36, 747-766, 1999.

[17] Das K. P., "A Mathematical study of a predator-prey dynamics with disease in predator", ISRN Applied Mathematics, 2011, 1-16, 2011.

[18] Haque M. and Venturino E., "An ecoepidemiological model with disease in predator: the ratio-dependent case", Mathematical Methods in the Applied Sciences, 30, 1791-1809, 2007.

[19] Brauer F. and Soudack A.C., "Stability regions and transition phenomena for harvested predator-prey systems", J. Math. Biol., 7, 319-337, 1979.

[20] Brauer F. and Soudack A.C., "Stability regions in predator-prey systems with constant-rate prey harvesting", J. Math. Biol., 8, 55-71, 1979.

[21] Dai G. and Tang M., "Coexistence region and global dynamics of a harvested predator-prey system", SIAM J. Appl. Math., 58, 193-210, 1998.

[22] Myerscough M.R., Gray B.F., Hogarth W.L. and Norbury J., "An analysis of an ordinary differential equation model for a two-species predator-prey system with harvesting and stocking”, J. Math. Biol., 30, 389-411, 1992.

[23] Narayan K. L. and Ramacharyulu N. CH. P., "A Prey-Predator Model with an Alternative Food for the Predator, Harvesting of Both the Species and with A Gestation Period for Interaction", Int. J. Open Problems Compt. Math., 1 (1), June 2008.

[24] Bairagi N., Chaudhuri S. and Chattopadhyay J., "Harvesting as a disease control measure in an eco-epidemiological system - a theoretical study", Mathematical Biosciences 217, 134-144, 2009.

[25] Madhusudhan K. Reddy and Lakshmi Narayan K., "A Prey-Predator Model with an Alternative Food for the Predator and Optimal Harvesting of the Prey", Advances in Applied Science Research, 2 (4):451-459, 2011.

[26] Naji R. K. and Ibrahim H. A., "The impact of disease and harvesting on the dynamical behaviour of prey predator model", Iraqi Journal of science, 53(1), 130-139, 2012.

[27] Haque M. and Venturino E., "Increase of the prey may decrease the healthy predator population in presence of disease in the predator", HERMIS, 7, 38-59, 2006.

[28] Hirsch M. W. and Smale S., "Differential Equation, Dynamical System, and Linear Algebra", New York, Academic Press, 1974.

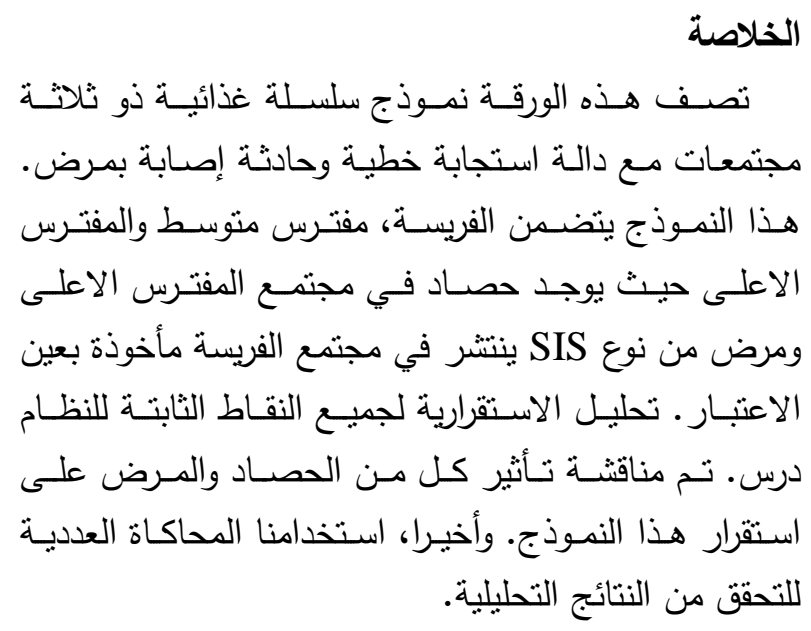

